\title{
Prediction of Strain Rate of Aluminum/Silicon Carbide Using Gray Level Run-Length Matrices
}

\author{
Ahmad E. Eladawi ${ }^{1}$, Saad A. A. Sayed ${ }^{2}$, Hammad T. Elmetwally ${ }^{3}$ and Tamer O. Diab ${ }^{4}$ \\ 1. Department of Mechanical Engineering, Benha University, Banha 13511, Egypt \\ 2. Department of Mechanical Design, Helwan University, Helwan 11731, Egypt \\ 3. Department of Mechanical Engineering, Beni-Suef University, Beni-Suef 62511, Egypt \\ 4. Department of Electrical Engineering, Benha University, Banha 13511, Egypt
}

Received: February 07, 2017 / Accepted: February 17, 2017 / Published: May 31, 2017.

\begin{abstract}
The microstructural processes occurring in metals and alloys during hot deformation are: DRX (dynamic recrystallization), superplastic deformation, dynamic recovery, wedge cracking, void formation, inter-crystalline cracking, prior particle boundary (FFB) cracking, and flow instability processes. Deformation characteristics of materials are interpreted as follows: in the low temperature (T $\leq 0.25$ of melting temperature) and high strain rate regime of 10 to $100 \mathrm{~s}^{-1}$, void formation occurs at hard particles and leads to ductile fracture. Many researchers used the currently defined statistical approaches to characterize images and extract useful information from the captured images. For more suitable of specific tasks, some researchers are introducing new texture features. HOS (higher-order statistics) estimate properties of three or more pixels occurring at specific locations relative to each other. GLRLMs (gray level run-length matrices) are popular method of HOS to extract texture features. This paper deals with texture features of GLRLM to predict strain rate values for Aluminum/Silicon Carbide.
\end{abstract}

Key words: Image processing, computer vision, GLRLMs, texture features, strain rate.

\section{Introduction}

Different microstructure defects are produced during the hot deformation process of the metal matrix materials due to the presence of the ceramic reinforcement material. The shape and the amount of these defects depend on the deformation conditions. Varma et al. [1] evaluated the microstructure and the fracture surface of aluminum reinforced with different particles sizes of $\mathrm{SiC}$ produced during tensile testing. Deformation characteristics of materials are interpreted as follows: in the low temperature $(T \leq$ 0.25 of melting temperature) and high strain rate regime of 10 to $100 \mathrm{~s}^{-1}$, void formation occurs at hard particles and leads to ductile fracture.

Corresponding author: Ahmad Elsaid Eladawi M., Ph.D., assistant professor, research field: using computer vision techniques in industrial.
With growing emphasis of industrial automation in manufacturing, vision techniques play an important role in many applications. One of the important applications of image processing analysis is texture analysis [2, 3]. Image texture, defined as a function of the spatial variation in pixel intensities (gray values), is useful in a variety of image processing applications and has been a subject of passionate study by many researchers. The meaning of the term texture, when image processing is concerned, is completely different from the usual meaning in the field of automated industries. In general, the process of texture analyzing requires the calculation of various features for each texture. Texture features contain information representative of visual characteristics, but also of characteristics which cannot be visually differentiated [4]. As different surfaces have different textures, the study of these textures forms an important cue for the 
recognition of surfaces. Texture analysis is the predominant method that plays an important role in many image processing applications, such as classification, segmentation, pattern recognition, etc. Because manufacturing involves machined surfaces, the study of textures of machined surfaces has become indispensable to the successful implementation of vision in manufacturing. Image texture can be quantitatively evaluated using some properties and various statistical and structural methods that have been developed to study these features. Statistical methods gained preference over structural methods, as most image textures do not follow a specific grammar or rule, which is essential for the successful implementation of structural methods [4]. The statistical methods have different orders based on the number of gray values considered analysis and the type of relationship used. The statistical methods involve calculation of properties based on the gray tones of the specimens [2].

\section{GLRLMs (Gray Level Run-Length Matrices)}

Some researchers still focus on introducing new texture features, which are more suitable for specific tasks [7]. HOS have been already involved in the texture analysis of the early years by Galloway [5]. These include interval cover and a series of statistics on the run-length methods. Tsatsanis and Giannakis [8] employed HOS to solve the invariant texture classification and modeling problems. GLRLM is a popular method introduced by Galloway [5] to extract texture features from images. GLRLMs are considered a higher-order statistical approach because it is used to characterize the spatial variation of pixel values in an image by three parameters: grey level of the run, the length of the run and the direction of the run. GLRLMs estimate the length of grey level runs. A grey level run is a connected set of pixels in a specific direction having the same grey values or differing from each other by a specified threshold. The length of the run is the number of pixel points within the run. In the GLRLMs, matrices are constructed in which each element denotes the number of runs of a particular grey level of a specified length. The matrix element $\mathrm{M}(i, j \mid \theta)$ of the grey-level run length matrix specifies the number of times a run of $j$ pixels occurs in the angle $\theta$ direction with pixel intensity of $i$. Four matrices can be constructed depending on the orientation of the runs $\left(0^{\circ}, 45^{\circ}, 90^{\circ}\right.$ and $135^{\circ}$ ). Unlike the GLCM, the run length matrix is not always a square matrix. To obtain numerical texture measures from matrices, Galloway [5] calculated five functions: SRE (short runs emphasis), LRE (long runs emphasis), GLNU (grey level non-uniformity), RLNU (run length non-uniformity) and RP (run percentage).

Finally, in the GLRLM approach, the gray level runs are characterized by the gray tone of the run and the length of the run and the direction of the run [6].

\section{Experimental Work}

Compression tests were performed using a computer controlled servo-hydraulic universal testing machine (Model UH-500 kNA, Schematize ${ }^{\mathrm{TM}}$, Japan), which had been modified to carry out uniaxial compression over a load capacity of 50 tons and a maximum ram speed of $50 \mathrm{~mm} / \mathrm{min}$. Each specimen was compressed between two grinded plates. The aluminum powders had an average grain size ranging from 80 to $250 \mu \mathrm{m}$. The silicon carbide particles had a grain size of $38 \mu \mathrm{m}$. The universal testing machine had a maximum load capacity of 50 tons. The machine also had a controlled electric muff furnace with a maximum temperature of $1,300^{\circ} \mathrm{C}$, which was used to heat the specimen to the required temperature.

All specimens were firstly heated to the required test temperature by a radiation furnace enclosing the test rig. The specimens were maintained at the required temperature for $5 \mathrm{~min}$ to ensure the uniform temperature distribution within the specimens. A thin layer of the burned oil with graphite was painted to the 


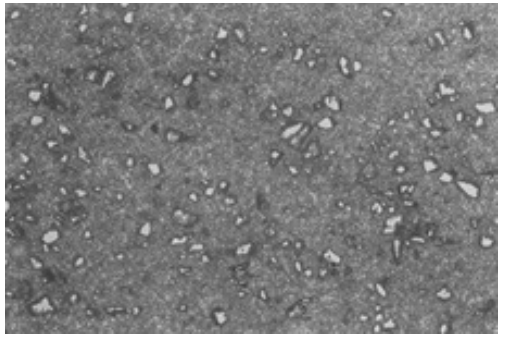

(a) $\mathrm{s}^{-1}=0.006$

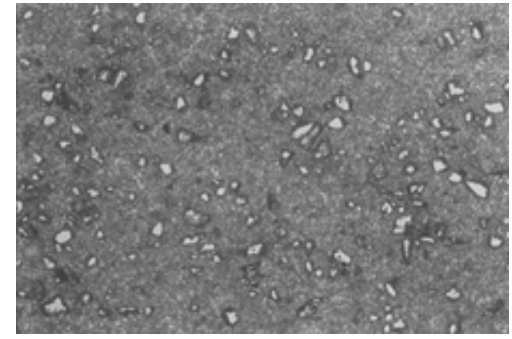

(b) $\mathrm{s}^{-1}=0.008$

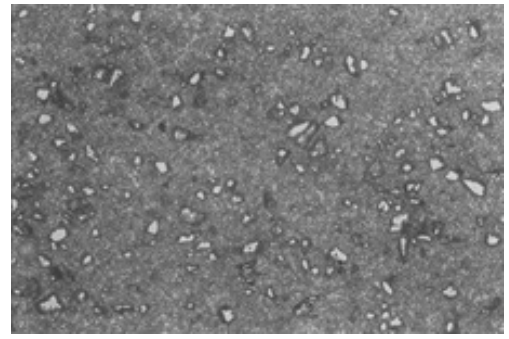

(c) $\mathrm{s}^{-1}=0.012$

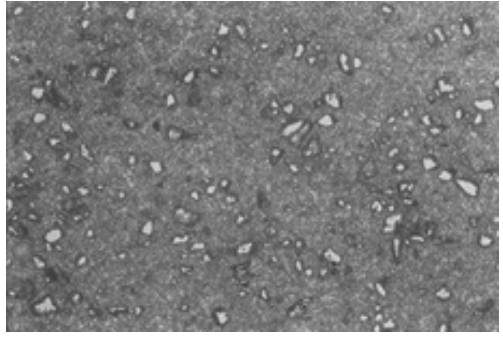

(d) $\mathrm{s}^{-1}=0.018$

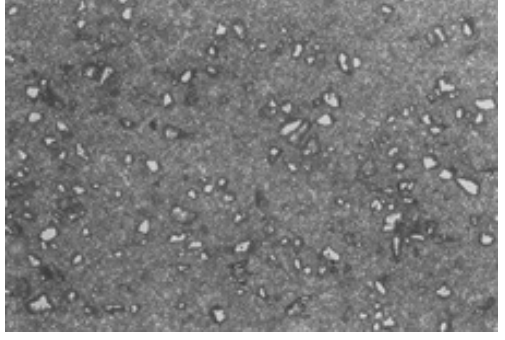

(e) $\mathrm{s}^{-1}=0.024$

Fig. 1 Surfaces of $\mathrm{Al} / \mathrm{SiC}$ samples with different strain rates.

plates to reduce the friction effect. The specimens were compressed to $60 \%$ of their initial height. The work was done with $450{ }^{\circ} \mathrm{C}$ in temperature and a range of strain rate between 0.006 and $0.024 \mathrm{~s}^{-1}$. To capture images, a JENAVERT incident-light research microscope with a high resolution digital camera was used. Fig. 1 illustrates some used sample surfaces with different forming strain rates.

\section{Used Software}

In this work math-lab program was used. It is helpful to use MATLAB Image Processing Toolbox. There are five types of images in MATLAB, and this work used Grayscale image type. A grayscale image $\mathrm{M}$ pixels tall and $\mathrm{N}$ pixels wide is represented as a matrix of double datatype of size $\mathrm{M} \times \mathrm{N}$. Element values denote the pixel grayscale intensities. In 2000, MATLAB was rewritten to use a newer set of libraries for matrix manipulation [9]. MATLAB was first adopted by researchers and practitioners in control engineering, Little's specialty, but quickly spread to many other domains. It is now also used in education, in particular the teaching of linear algebra, numerical analysis, and is popular amongst scientists involved in image processing [10]. Through this work, an interface named "STRGL software" was created to deal with the relationship between the features and changes in strain rate through the experimental work. Fig. 2 represents a program interface. The interface gives the option to open a selected image and calculates its properties as illustrated in Fig. 2. The interface gives the texture features values for the opened image. The calculated data were used in MS Excel to get the resulting curves for every feature in different cases according to changes in temperature.

\section{Results and Discussions}

The results showed that the changes in structure

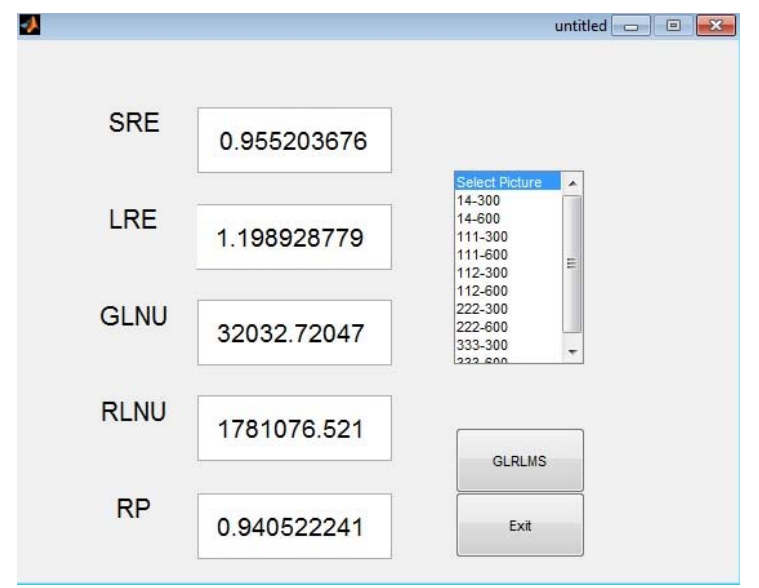

Fig. 2 Interface of STRGL program. 
cause changes in the texture features values and its sensitivity according to the changes in strain rate. Figs. 3-7 represent these results. Strain rate values are represented in the horizontal axis in every curve.

From the resulting curves, 4 texture features have high sensitivity (more than $90 \%$ ) to changes in strain rate through the experimental work. The sensitive feature can be illustrated in the next curves.

The correlation coefficients for sensitive features are illustrated in Table 1.

The previous curves and table give the next observation:
- SRE, LRE, GLNU and RP features are the highest sensitivity features for the change in strain rate.

- SRE, LRE, GLNU and RP features have good correlations with the change in strain rate.

- High sensitivity curves give the ability to observe the trend of every curve, and so, to predict the strain rate values.

- RLNU feature is a poor sensitive feature for the change in strain rate. RLNU feature has a correlation coefficient of 0.8613 . So, it cannot be used to study these changes.

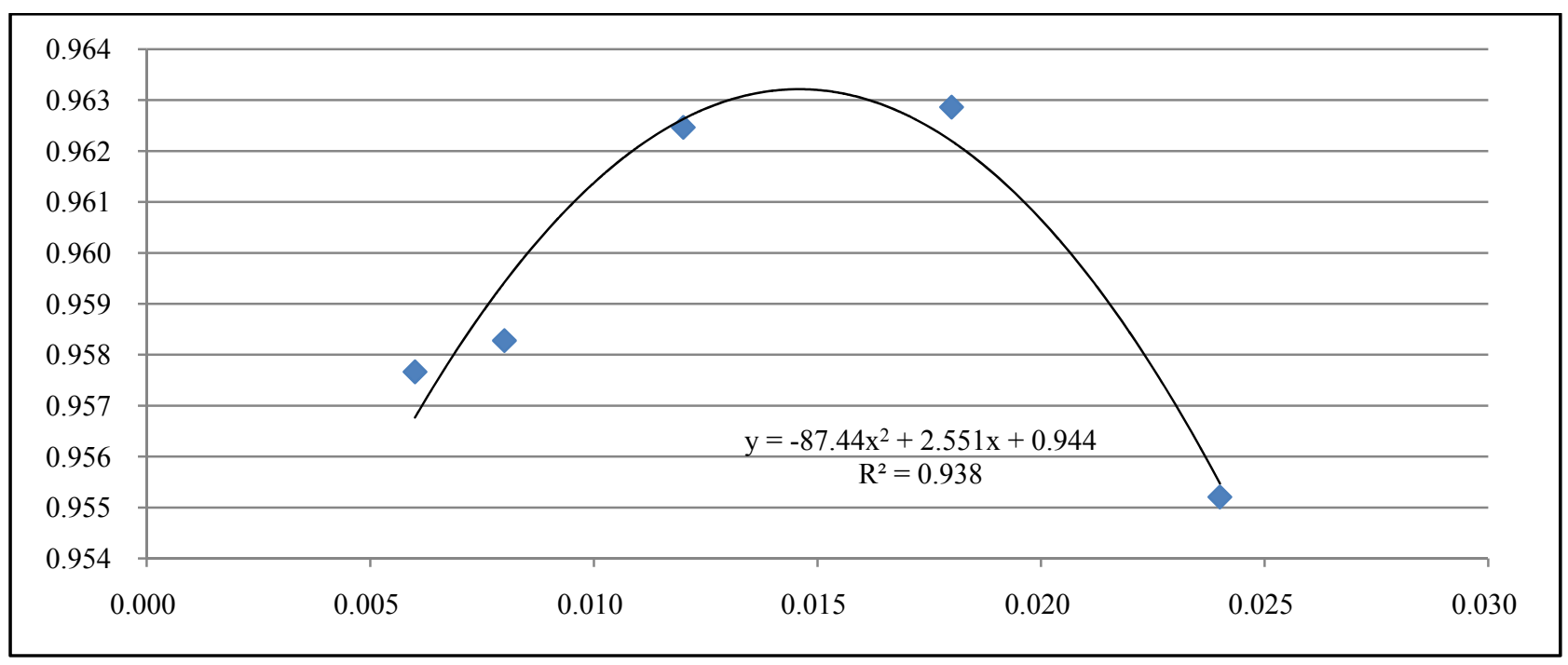

Fig. 3 SRE/Strain rate curve.

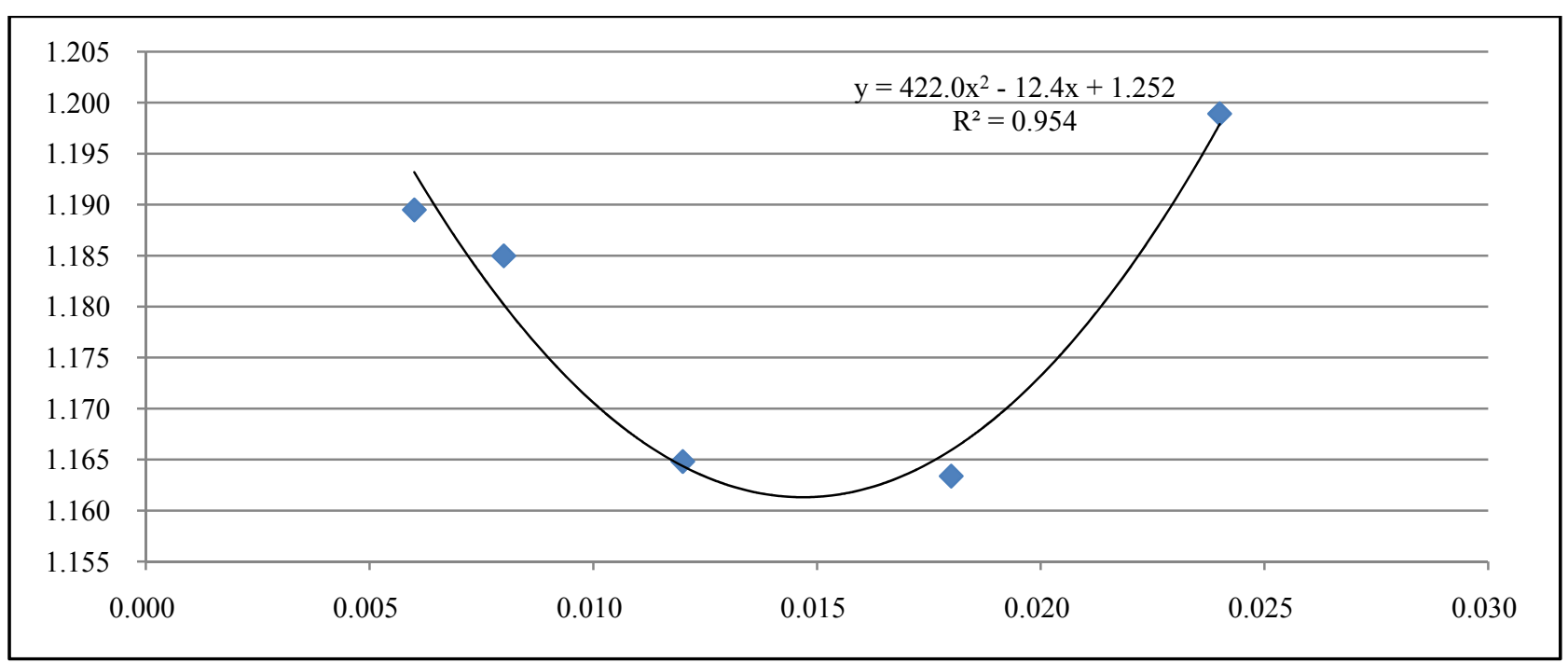

Fig. 4 LRE/Strain rate curve. 


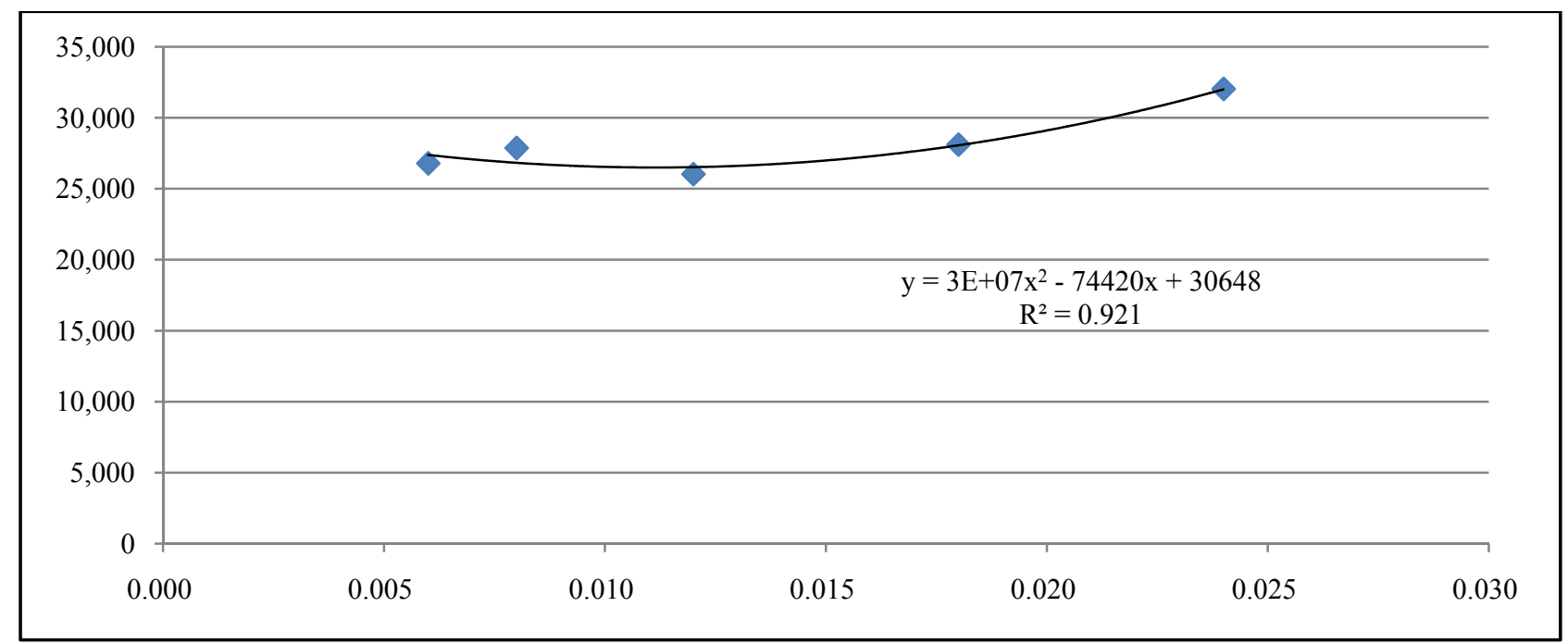

Fig. 5 GLNU/Strain rate curve.

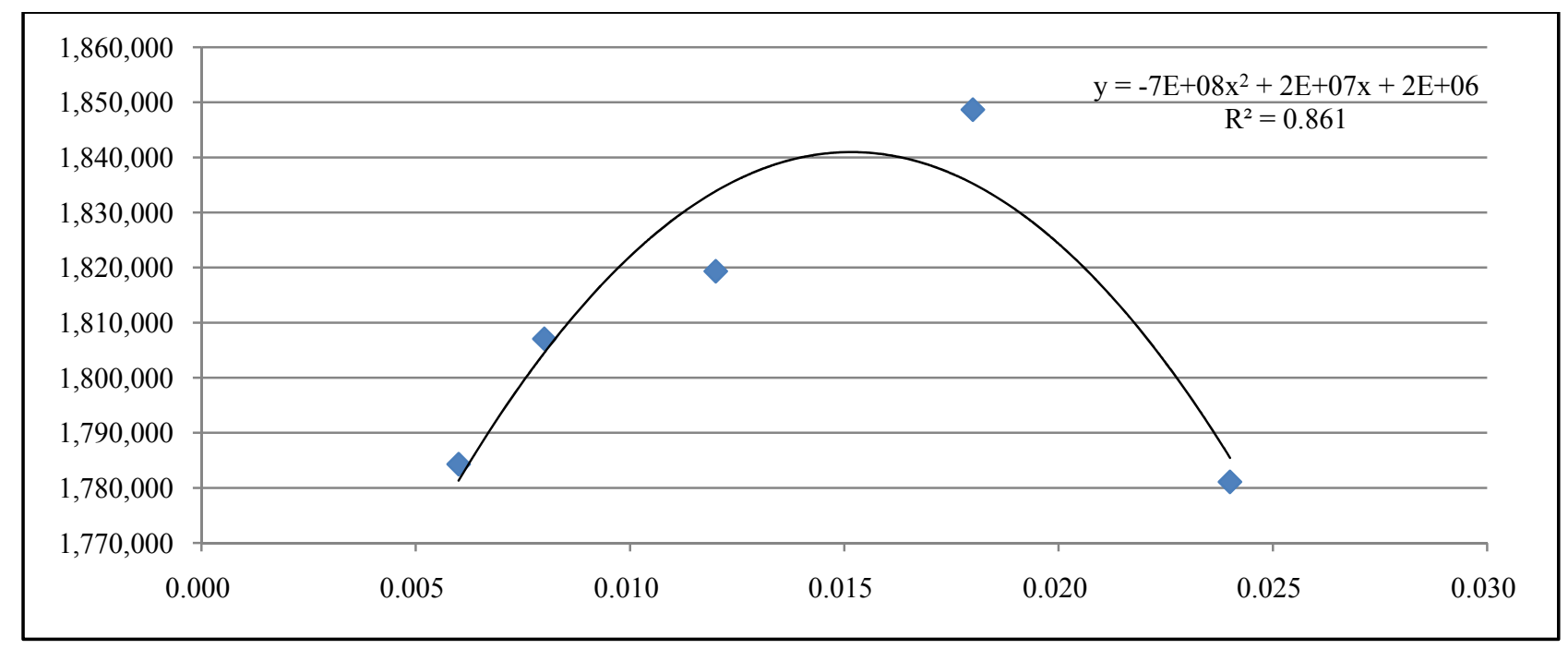

Fig. 6 RLNU/Strain rate curve.

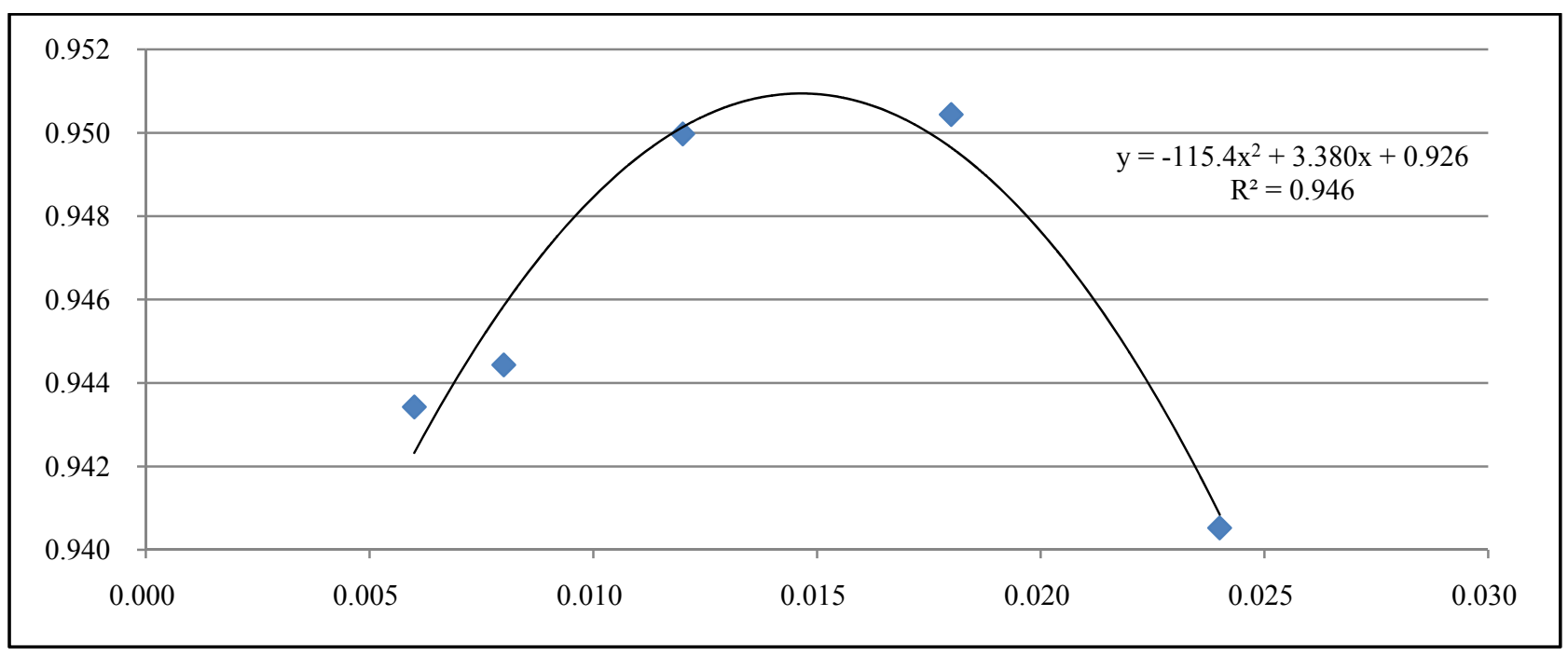

Fig. 7 RP/Strain rate curve. 
Table 1 Correlation coefficients of sensitive texture features versus strain rate values.

\begin{tabular}{lllll}
\hline Texture feature & SRE & LRE & GLNU & RP \\
\hline Correlation coefficient & 0.9386 & 0.9548 & 0.9217 & 0.9463 \\
\hline
\end{tabular}

\section{Conclusions}

The shape and the amount of defects of the surfaces of aluminum reinforced by $10 \mathrm{wt} . \% \mathrm{SiC}$ depend on the deformation conditions. In the present work, the effects of strain rate on the forming behavior of aluminum reinforced by $10 \mathrm{wt} . \% \mathrm{SiC}$ were studied by using image texture features after using the compression test which was done at constant temperature as $450{ }^{\circ} \mathrm{C}$ and a range of strain rate between 0.006 and $0.024 \mathrm{~s}^{-1}$. This paper studied the relationship between GLRLM features and changes in operation strain rate of $\mathrm{Al} / \mathrm{SiC}$ materials. The results showed that 4 texture features have high coefficient correlations with changes in strain rate, but one feature has a poor sensitivity to these changes. The sensitive results give the ability to predict the strain rate values for the same materials and temperature.

\section{References}

[1] Varma, V. K., Kamat, S. V., and Kutumbarao, V. V. 2001. "Tensile Behavior of Powder Metallurgy Processed (Al-Cu-Mg)/SiCp Composites." Materials Science and Technology 17 (1): 93-101.

[2] Venkatramana, K., and Ramamoorthy, B. 1996. "Statistical Methods to Compare the Texture Features of Machined Surfaces." Pattern Recognition 29 (9):
1447-59.

[3] Haralick, R. M., Shanmugam, K., and Dinstein, I. 1973. "Textural Features for Image Classification." IEEE Transactions on Systems, Man and Cybernetics SMC-3 (6): 610-21.

[4] Bharathi, P. T., and Subashini, P. 2013. "Texture Feature Extraction of Infrared River Ice Images Using Second-Order Spatial Statistics." World Academy of Science, Engineering and Technology International Journal of Computer, Electrical, Automation, Control and Information Engineering 7 (2): 747-57.

[5] Galloway, M. M. 1975. "Textural Analysis Using Gray Level Run Lengths." Computer Graphic and Image Processing 4 (2): 172-9.

[6] Tsatsanis, M. K., and Giannakis, G. B. 1992. "Object and Texture Classification Using Higher Order Statistics." IEEE Trans. PAMI 14 (7): 733-50.

[7] Gadelmawla, E. S., Koura, M. M., Maksoud, T. M. A., Elewa, I. M., and Soliman, H. H. 2002. "Roughness Parameters." Journal of Materials Processing Technology 123 (1): 133-45.

[8] Moler, C. 2000. "MATLAB Incorporates LAPACK." Cleve's Corner. MathWorks. Retrieved 20 (December 2008).

[9] Moler, C. 2007. "The Origins of MATLAB.” (December 2004 Retrieved 15 April 2007).

[10] Navas, K. A., and Jayadevan, R. 2014. "Lab Primer Through MATLAB®: Dgital Signal Processing, Digital Image Processing, Digital Signal Processing And Digital Commiication." PHI Learning Private Limited, Delhi-110092. 


\section{Appendix A}

The next table illustrates the different texture features as definitions and equations:

\begin{tabular}{|c|c|c|}
\hline Feature & Definition & Equation \\
\hline SRE (Short run emphasis) & $\begin{array}{l}\text { SRE indicates the number of the short runs } \\
\text { in the GLRLM. }\end{array}$ & $\mathrm{SRE}=\frac{\sum_{k}^{n} \sum_{l}^{m} \frac{M_{m}(k, l)}{l^{2}}}{\sum_{k}^{n} \sum_{l}^{m} M_{m}(k, l)}$ \\
\hline LRE (Long run emphasis) & LRE indicates the number of the long runs. & $L R E=\frac{\sum_{k}^{n} \sum_{l}^{m} M_{m}(k, l) * l^{2}}{\sum_{k}^{n} \sum_{l}^{m} M_{m}(k, l)}$ \\
\hline GLNU (Gray level non uniformity) & $\begin{array}{l}\text { GLNU gives a description of the } \\
\text { distribution of the gray level. }\end{array}$ & $G L N U=\frac{\sum_{k}^{n}\left(\sum_{l}^{m} M_{m}(k, l)\right)^{2}}{\sum_{k}^{n} \sum_{l}^{m} M_{m}(k, l)}$ \\
\hline RLNU (Run length non uniformity) & $\begin{array}{l}\text { RLNU refers to the distribution of the pixel } \\
\text { run length. }\end{array}$ & $R L N U=\frac{\sum_{l}^{m}\left(\sum_{k}^{n} M_{m}(k, l)\right)^{2}}{\sum_{k}^{n} \sum_{l}^{m} M_{m}(k, l)}$ \\
\hline RP (Run percent) & RP describes the grainy of images. & $R P=\frac{\sum_{k}^{n} M_{m}(k, l)}{\sum_{k}^{n} \sum_{l}^{m} l M_{m}(k, l)}$ \\
\hline
\end{tabular}

